\title{
Transelectric's Transmission Control Center (CCT): Functionality and Development Strategy
}

\author{
Roberto Carrillo Calderón, Project Coordinator \\ National Energy Transmission Company - TRANSELECTRIC \\ 6 de Diciembre N26 - 235 Quito, Ecuador \\ email: rcarrillo@transelectric.com.ec
}

\begin{abstract}
The objective of this paper is to report on the background, the relevant characteristics and the strategy adopted by TRANSELECTRIC for the development of its Transmission Control Center (CCT), a fundamental element to put into practice the foreseeing operative philosophy and to efficiently comply with current regulations, since the Ecuadorian Electrical Sector was restructured to a deregulated and competitive market.
\end{abstract}

The CCT project is part of the automation of the National Transmission System of TRANSELELCTRIC, which includes two additional and complementary projects, namely: the Modernization of Substations and the Strengthen of the Communication System with fiber optics. The aim is oriented to the improvement of the service quality, to reduce costs and to accomplish with all the requirements derived from the structure of the Electrical Sector. This paper refers to the CCT.

Since the CENACE (National Energy Control Center Corporation) is actually in the process of updating its Energy Management System, a common strategy was established with them for the development of the CCT

The supply for the CCT includes the provision of hardware and software for the real time operation, for the preventive and corrective security analysis of the National Transmission System operation, for the maintenance and administrative work.

The services to be provided by the supplier are oriented to guarantee the technical capacity of TRANSELECTRIC for the operation of the CCT under normal and fault conditions, for the maintenance and programs development, and for the use of the CCT as an Information System for a variety of technicalmanagerial tasks.

The Energy Management System (EMS) is supplied by ABB Inc. Company from U.S.A. The contract was signed in March 2004. The System will be in service by the beginning of the year 2006 .

The civil work, the auxiliary services and the communication facilities for the EMS appropriate environment demanded a big effort and a close coordination among suppliers.

This paper is based on TRANSELECTRIC experience in the development of the CCT project, as well as in the Company strategies for the development of other related projects.

\section{DRIVING FORCES}

The Law and the Regulations for the Electrical Sector establishes rigorous requirements of security and service quality, which forces to have tools, as it is the case of the CCT, that allow to satisfy them, maintaining suitable economic indices.

One of the important operative objectives of TRANSELECTRIC is to obtain, in the medium term, the automated and remote management of its substations. Under those circumstances, the CCT will be required for the unattended real time operation, as well as for the pre and post operative analyses.

Additionally it is necessary to reinforce the technical and administrative management of TRANSELECTRIC and, particularly, the one of maintenance, for which it is required to centralize a big amount of information from each one of the maneuver, measurement and protection elements of the substations.

\section{BACKGROUND}

The former Ecuadorian Electrification Institute (INECEL) was in charge of the Planning, Construction and Operational Control of the Interconnected National System (Generation and Transmission). For this purpose it had, since 1994, an Energy Management System (EMS) named SPIDER, from ABB Sweden.

The Law for the Electrical Sector, effective in 1996, established the desegregation of INECEL in generation companies, one transmission company (TRANSELECTRIC) and the CENACE.

The above EMS became property of CENACE. One console of this EMS has been used in remote mode by TRANSELECTRIC, with important limitations.

\section{AUTOMATION OF THE NATIONAL TRANSMISSION SYSTEM}

TRANSELECTRIC is executing, besides the Transmission Control Center (CCT), two complementary projects: one is the Substations Modernization and the other is the Telecommunications Reinforcement with fiber optics. The development of the CCT project becomes a key factor to guarantee consistency and harmony among them. 
These projects have as objectives the service quality improvement (voltage levels and frequency, continuity of the service), the costs reduction (operation, maintenance, deferment of investments) and the fulfillment of the standards established within the framework of the Wholesale Electrical Market MEM (that means the interaction with the agents of the Market, the CENACE and the Regulating Entity).

The National Interconnected Network operative and maintenance requirements imposes technical and technological improvements for Network real time analysis, supervision and control, as well as a close centralized wide view of each substation parameters and elements behavior.

\section{A. Telecommunications Reinforcement}

The Telecommunications Reinforcement project is oriented to assure the present and future communications requirements for TRANSELECTRIC, the CENACE and the agents of the MEM. This project is in execution, by stages. The CCT and the CENACE Control Center depend on this project, so that they can work with their full functionality.

The telecommunications system will consist on a redundant data transmission system supported by PLC and fiber optics. The fiber optics will be basically installed over the $230 \mathrm{kV}$ ring lines and on the extensions towards the two borders (Colombia and Peru transmission systems). PLC (Power Line Carrier) is the communication media that will be used in most radial links in order to complement the TRANSELECTRIC's communication system. PLC digitalization is foreseen for some links.

\section{B. Substations Automation}

The Substation Automation Project is oriented to optimize the local and remote operative handling and the maintenance management for TRANSELECTRIC substations. This effort requires a process of technological modernization. One of the fundamental goals is to make it possible the unattended operation of the substations, from the CCT.

Other goals are related with more visibility for improved local operation and for maintenance, with the technological upgrade according to needs, with the best practices and with the market state of the art.

This long term project (planned to be concluded in 5 years from now), contemplates the modernization of up to 30 substations. It has begun this year with a pilot project and a basic study It will be continued with a deeper cost - benefit analysis and with an staggered implementation.

\section{CCT PROJECT SCOPE}

The CCT is an Energy Management System - EMS and includes the following functionalities: data acquisition and supervisory control (SCADA), network security functions, development and maintenance, file and information retrieval, and simulation for training. The Control Center will be connected initially with around 30 substations, the majority of them with old protection and control technologies. In addition it will give support to diverse operational, maintenance and executive functions.

The CCT supply corresponds to the provision of the necessary hardware and software to fulfill the requirements of the real time operation, pre and post operative analysis, development and maintenance.

The contracted services includes the detailed design, the installation, subsystems integration, the factory tests and site tests, the documentation production, training and coordination of the participation of the TRANSELECTRIC's personnel during all the stages of the project, so they will be able to operate and maintain the CCT.

The project included the infrastructure required for the installation and operation of the EMS: civil work, auxiliary services, and a fiber optic communication link.

\section{BENEFITS}

The CCT SCADA and Network Applications functionalities gives tangible benefits, including the increase of the Power System reliability, the control and the energy losses reduction, the potential delay in the investments and the improvements in programmed maintenances. The benefit-cost analysis was conducted, and gave as a result a positive rate of over 2.7 .

The project has also a series of qualitative or intangible benefits, such as the monitoring improvements, the data collection and reports generation, the better coordination and supervision of the operation of the National Transmission System, the optimized use for operation and maintenance of the personnel allocated in the substations and, which is more relevant, the improvement of the energy service quality.

As mentioned before, the Law and the Regulation for the Electrical Sector establishes rigorous 
requirements of security and service quality. For this reason, the CCT becomes one of TRANSELECTRIC's strategic tools that will allow the compliance of above requirements and will give the technical support for Agents needed information and disputes.

\section{ARCHITECTURE AND FUNCTIONALITY}

\section{A. Functional Components}

The CCT has been conceptualized on the basis of the following components:

- Energy Control System, that includes SCADA functionality and network applications

- Storage and Data retrieval System.

- Project Data Development and programs Maintenance System (PDS).

- Dispatcher Training Simulator (option).

\section{B. EMS Functionality}

- $\quad$ Energy Control System (ECS):

The ECS is the real-time nucleus of the EMS. The ECS is a high-availability and high performance system characterized by high-speed data collection, presentation and control functions.

A Power Network Analysis - including State Estimator, Power Flow Studies, Optimal Power Flow, Equipment Outage Scheduling, Contingency Analysis and a Message Exchange function are components of the ECS.

A web server is implemented for information visualization, via internet, for selected users.

- Information Storage and Retrieval System (IS\&R):

Historical Information Management, Study Save Cases Management, System Message Log Storage and Retrieval, and Disturbance Data Collection functions conform the IS\&R.

The IS\&R functions execute on a platform dedicated to that functionality. A compression algorithm allows saving information nearly with a SCADA time resolution and for a long time periods.

The IS\&R supports EMS users and a number of nonEMS users. In this sense it is the link between the EMS and other corporate needs.
- Project Data Development and programs Maintenance System (PDS):

The PDS is a compacted system that includes almost all the ECS functionality. It is intended to be used at Site for diverse project development needs, from the beginning of the project (in parallel with the Factory system development) and later for maintenance purposes.

\section{- $\quad$ Dispatcher Training Simulator (DTS):}

The DTS supports the EMS functionality and it is able to simulate the power system steady state condition. It has the required facilities for the teacher - students interaction. The DTS will be used by TRANSELECTRIC on a trial basis, during one year, before the decision of buying it.

\section{CCT Architecture}

The EMS is a redundant, distributed computing environment using Local and Wide Area Networks (LANs and WANs) to interconnect the system components and employs industry standards for hardware, software, and user interfaces. This type of "open" architecture allows the addition of future functionality and the replacement of hardware without disruption to the EMS. The conceptual EMS configuration is illustrated in figures 1 and 2 .

The components and basic architecture can be visualized in figure 1

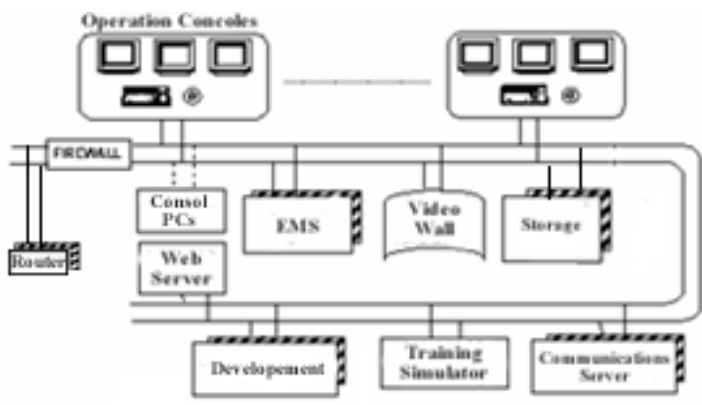

Fig 1: Components and Architecture

\section{Remote Data Acquisition}

The CCT counts on interfaces with Remote Terminal Units or data concentrators at substations, with Remote Frond End data concentrators, with connected remote consoles, with the CENACE's Control Center, with operations centers of generating and distributing agents for information exchange at 
computer-computer level, and with the Information System of TRANSELECTRIC.

The CCT data acquisition reuses the existing remote equipment (RTUs of property of the CENACE) and some gateways at the new automated substations. The Substations Automation project is executed with new technologies, those that will allow a more efficient form of data acquisition and control, so that the substations, in the future, could be operated unattended from the Transmission Control Center.

As part of the joint effort with the CENACE, concentrators of remote data have been installed (front - ends) to handle groups of RTU's so that communications could be optimized.

CENACE is the proprietary of the Front Ends. Therefore, the implementation of these elements is managed by $\mathrm{ABB}$ Inc. and CENACE.

A partial back up concept is possible to adopt between both control centers for emergency conditions, since most data acquisition facilities are common (data communications media, front ends and RTU's) and the supplier is the same for the control centers, with similar functionality.

Figure 2 shows the scheme of data transfer from/to TRANSELECTRIC, from/to its own substations, the agents' substations / control centers and the CENACE. The final configuration considers four redundant front ends, located in four substations, according to the National Transmission System communication structure.

As it can be seen from the figure 2, the Front Ends are crucial for Control Centers SCADA functionality.

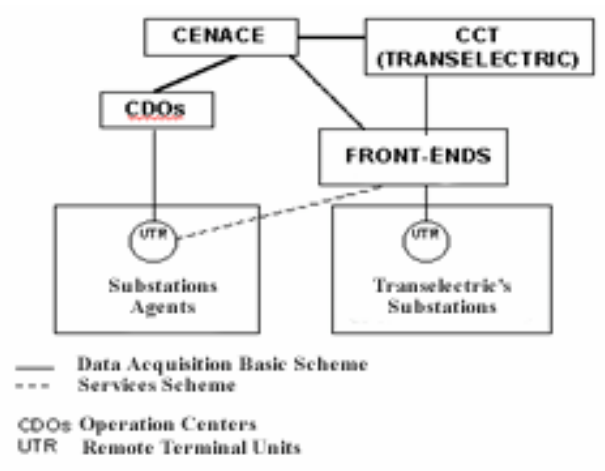

Fig 2: Data Acquisition Scheme

VII. PROJECT DEVELOPMENT STRATEGIES

A. Preliminary activities
A development group for the project, within TRANSELECTRIC, was conformed. The basic definitions were outlined, supported with technicians of the diverse areas that will be the users of the CCT. In this way it was prepared the first engineering specifications that served as a departure point for the technical specifications.

Then, an inter-institutional (TRANSELECTRIC CENACE) group was created which took the responsibility, with the cooperation of KEMA, a consulting firm, to carry out the studies, the call for bids documents and the final specifications for the implementation of the Transmission Control Center (CCT) and of the Updating the CENACE's Energy Control Center.

\section{B. Bidding}

TRANSELECTRIC made an international bidding for Supply and Installation of the CCT. The selection method included a combination of technical and economic aspects, on the basis of a model prepared for this purpose, in which the weights corresponding to the different parameters for goods and services are defined. The model considers also the costs associated to maintenance, in order to evaluate the potential whole costs during the System lifetime.

The Energy Management System supplier is ABB Inc. Company from U.S.A. The contract was signed in March 2004. The product is named Network Manager (former RANGER).

Independent calls for bids (CENACE and TRANSELECTRIC) processes were decided and the required coordination for common needs is in progress.

\section{Project schedule)}

All the activities for the project implementation will last 22 months. The guaranty period will be 3 years (for hardware) and one year (for software), starting after the System final acceptance.

Detailed design activities and basic training was conducted at Site, at the beginning of the project.

The system factory development, training and tests started in August 2004 and were completed during the first semester of the year 2005.

Some unforeseen delays in the CCT building and in the shipping from the supplier headquarters gave as a result that the installation process started at the beginning of November, 2005. Two months site tests will be required. 
Point to point tests for some TRANSELECTRIC substations were rescheduled due to Front Ends stabilization process. Pending substations point to point tests will be done shortly, in this year.

The System will be in service at the beginning of January, 2006. Forty five days availability test will be conducted before the final acceptance.

\section{Project Development System (PDS)}

The PDS was used for database and display adjustments, as well as for code and point to point substation elements test. It will be used during EMS operation for database maintenance and for software development.

Point to point tests, conducted in parallel with the corresponding CENACE EMS tests, lasted about four months and represents about $60 \%$ of total required point to point tests. Since the EMS system is already installed, point to point tests with remaining substations will be conducted with this system instead.

These activities had been in progress in parallel with some factory CCT development activities and with required auxiliary services implementation and civil work. In this way, project delays were reduced.

\section{E. CIM Tool Kit (future)}

The implementation of the Common Information Model (CIM) tool kit for improved EMS maintenance was postponed. This implementation will be done depending on the type of maintenance contract to be signed with the supplier and on the TRANSELECTRIC's analysis of the benefits of this tool comparing with the associated hardware, basic software and training costs.

\section{F. Complementary aspects}

- TRANSELECTRIC participated actively in the development of the project, through their technical personnel, with the support of external consultants. In this way, the human resource for the stage of operation and maintenance of the CCT was empowered. An interdisciplinary team was formed (power system, electronics and information system engineers formed the basic development group.

- It was decided to adapt the areas of one of the TRANSELECTRIC buildings, in which the CCT is being installed. The required high availability auxiliary services have been implemented (power supply, diesel generator, UPS, air conditioning, fire detection and protection system, security monitoring, access control, auxiliary services centralized supervision, etc.). Main auxiliary systems indications will be sent to the Network Manager using DNP 3.0 protocol.

A close coordination among contractors was required, in order to allow a proper and on time environment for the EMS. In particular, the requirements for the operation room BARCO VideoWall (approx. $2.60 \mathrm{~m}$ x $4.00 \mathrm{~m}$.) needed a careful implementation.

- $\quad$ EMS operators and all operative engineers will be moved from their actual offices (TRANSELECTRIC main office building) to the CCT building. Appropriate logistics for new location is in progress.

- A five $\mathrm{km}$. fiber optics link was implemented between one substation and the CCT building for SCADA and corporate needs. A $2 \mathrm{Mbps}$ channel is available for SCADA and another 2 Mbps is available for ICCP, supplier remote maintenance, corporate information system network, telephone lines, and others. A redundant communication link is foreseen shortly.

\section{CONCLUSIONS}

- The CCT is conceptualized for the real time operation, preventive and corrective analysis of the operation of the National Transmission System, and for the support to the maintenance and some administrative management tasks.

- One of the fundamental goals of the project is to make possible, in the near future, the unattended operation of the substations, from the CCT.

- The Law and the Regulation for the Electrical Sector establishes rigorous requirements of security and service quality, The CCT is a fundamental System in order to satisfy them.

- The services given by the contractor were oriented to guarantee the technical capacity of TRANSELECTRIC for the operation and maintenance of the CCT.

- The benefit-cost analysis threw a relation of over 2.7. In addition, relevant qualitative benefits exist.

- $\quad$ The CCT Project will take advantage of synergies with the projects for Substations Automation and Telecommunications Reinforcement with fiber optics. 
- The Front End computers are critical elements in the common CENACE - TRANSELECTRIC data acquisition architecture.

- For the pre bid documents and for the implementation process it was implemented a joint strategy with the CENACE (for common needs).

- The EMS purchased system was bought to ABB Inc. and it is named Network Manager (former RANGER).

- Specialized support for the project development was contracted with KEMA Consulting.

- TRANSELECTRIC has implemented a sub project for the required facilities (civil work and auxiliary services) and a close coordination among suppliers was needed.

- The CCT will be in commercial operation at the beginning of the year 2006 . 